Max-Planck-Institut für demografische Forschung

Max Planck Institute for Demographic Research

Doberaner Strasse $114 \cdot$ D-18057 Rostock $\cdot$ GERMANY

Tel +49 (0) 3812081 - 0; Fax +49 (0) 3812081 - 202;

http://www.demogr.mpg.de

MPIDR WORKING PAPER WP 2002-018

MAY 2002

\title{
The cultural evolution \\ of age-at-marriage norms
}

Francesco C. Billari (billari@demogr.mpg.de)

Alexia Prskawetz (fuernkranz@demogr.mpg.de)

Johannes Fürnkranz (juffi@ai.univie.ac.at)

(C) Copyright is held by the authors.

Working papers of the Max Planck Institute for Demographic Research receive only limited review. Views or opinions expressed in working papers are attributable to the authors and do not necessarily reflect those of the Institute. 


\title{
The cultural evolution of age-at-marriage norms
}

\author{
Francesco C. Billari \\ Max Planck Institute for Demographic Research, \\ Doberaner Str. 114, D-18057 Rostock, Germany \\ E-mail: billari@demogr.mpg.de \\ Alexia Prskawetz \\ Max Planck Institute for Demographic Research, \\ Doberaner Str. 114, D-18057 Rostock, Germany \\ E-mail: fuernkranz@demogr.mpg.de \\ Johannes Fürnkranz \\ Austrian Research Institute for Artificial Intelligence \\ Schottengasse 3, A-1010 Wien, Austria \\ E-mail: juffi@ai.univie.ac.at
}

March 11, 2002

\begin{abstract}
We present an agent-based model designed to study the cultural evolution of age-at-marriage norms. We review theoretical arguments and empirical evidence on the existence of norms proscribing marriage outside of an acceptable age interval. Using a definition of norms as constraints built in agents, we model the transmission of norms, and of mechanisms of intergenerational transmission of norms. Agents can marry each other only if they share part of the acceptable age interval. We perform several simulation experiments on the evolution across generations. In particular, we study the conditions under which norms persist in the long run, the impact of initial conditions, the role of random mutations, and the impact of social influence. Although the agent-based model we use is highly stylized, it gives important insights on the societal-level dynamics of life-course norms.
\end{abstract}




\section{Introduction}

Normative guidelines matter when individuals make decisions about important events in their life. Marriage and childbearing constitute examples of such key events. The persistence of norms concerning demographic events in contemporary societies is nevertheless somehow surprising to some scholars who would expect a natural expansion of individuals' freedom also in the realm of demographic choices. As Livi Bacci observes for Europe, the long-run evolution of population is a movie in which the forces of choice are in the course of winning against the forces of constraint [21].

Agent-based modelling can provide us with useful insights into how norms constraining the demographic choices of individuals can persist in the longrun and survive for several generations. Relatively scarce empirical evidence on the existence of age-related norms, at least in contemporary societies, and the debate on the possibly decreasing relevance of norms over historical time, have so far limited the attention of scholars towards this topic. For instance, there is no widely agreed definition of norms affecting choices in the life course [27]. Agent-based modelling and social simulation approaches have, on the other hand, given very interesting results on norms.

In this paper, we propose to study the dynamics of age-at-marriage norms using an agent-based model. We focus on the evolution of norms, with the idea that "evolutionary perspectives can enhance our understanding of human demography", as Wilson [28] noted. Our view is that the long-term persistence of norms restricting life-course choices cannot be taken for granted.

The paper is structured as follows. The background is sketched in Section 2. Starting from the importance of norms in life-course research, we review some empirical evidence based on survey data on the existence of age-at-marriage norms. We briefly illustrate various definitions in the literature and their im- 
plementation in agent-based modelling. In Section 3, we introduce the agentbased model we use in order to study the dynamics of age-at-marriage norms. We describe in particular the intergenerational transmission of norms, the intergenerational transmission of the transmission mechanism itself, and the way we implement social influence in the model. Section 4 discusses various experiments we conducted using the model, and the implication of these experiments in the light of their demographic interpretation. In particular, we study the conditions under which norms persist in the long run, the impact of the initial distribution of the population, the impact of random mutations, and the importance of social influence. Finally, Section 5 draws a summary of the main results, and it outlines some perspectives for further research.

\section{Background}

\subsection{Social norms and the life course approach}

The life course approach [16], which has been particularly influential in the study of demographic events, stresses the importance of norms in shaping the life of individuals. The existence of norms on life-course events is advantageous for the individual, since it provides a guide to decision-making in an otherwise very complex environment. An extreme case of decision-making is choosing in conformity to a norm without thinking about it, as Epstein [13] put it. Psychological advantages of normative reasoning may also explain why life-course norms need not necessarily be enforced by explicit sanctions in all situations. For instance, Heckhausen [17], states that social norms may have been internalized in our society, rendering obsolete any need for external societal enforcement of social norms. She concludes that "life-course patterns would be expected to have become increasingly regulated by internalized norms about age-appropriate 
behavior, age-graded events and transitions, and age-sequential rules (e.g. you must finish school first before you can have a family) as societal regulation became more lenient".

The overall idea of the existence of social norms affecting demographic events implies the emergence and the evolution of more or less ordered patterns at the societal level. Nevertheless, as Marini [22] has correctly pointed out, the mere observation of patterns on the age distribution of certain behaviors cannot be used as an argument for the existence of age-related norms. On the other hand, also in absence of a single visible behavioral pattern at the aggregate level, age norms may play a role in decision-making. This would happen if agerelated norms were differential characteristics of ethnically or socially separated groups - a situation which we may define diversity of norms. Norms would then only be observed if one were able to analyze such groups in a separate way.

Norms on demographic events may concern timing (mostly, the age at which events are experienced by individuals), sequencing (how demographic events are ordered in the life course), and quantum (how many demographic events are experienced) [4]. Norms may also refer to "should" (prescription) or "should not" (proscription) type of statements. In the case of age norms on marriage, for instance, there may be norms on the age at which an individual should get married, as well as norms on the ages before which (or after which) an individual should not get married. In what follows, we concentrate on norms of the "should not" type.

\subsection{Some empirical evidence on age-at-marriage norms}

Age-related social norms on life-course events are embedded in the overall social system, and they might also become explicitly written into a legal system, although normative and legal control are sometimes alternatives to each other 
[19]. The legal aspect of life-course norms is particularly evident for age at marriage: in virtually all countries in the world there is a minimum age before which individuals are not allowed to get married.

Surprisingly, not much empirical research has been conducted on the presence of age norms. Researchers have usually limited themselves to assuming the existence, or non-existence, of age norms - especially of the proscriptive type - rather than examining the issue. Recently, some empirical studies have explicitly started to address and describe the existence of proscriptive age norms, mainly starting from the work of Settersten and Hägestad [25]. Settersten and Hägestad used a survey of individuals belonging to different age groups in the population of Chicago, and they showed that $82.3 \%$ of the respondents perceived an age deadline for marriage, that is an age after which one ought not marry. In a different geographical context, Billari and Micheli [3] used a survey in Friuli-Venezia Giulia (a region in North-Eastern Italy), where women were interviewed about their perception of the existence of ages before which, or after which, one should not start partnerships. Some results from this survey are reported in Table 1. More detailed empirical results are thus still lacking, and this does not allow us to draw a complete picture. Nevertheless, we can safely take for granted that, nowadays, lower age limits (an age before which one ought not marry) for age at marriage are both embedded in the legal system and perceived within significant groups. The existence of upper age limits, or age deadlines may depend on the context and the population studied, and it needs further empirical investigation.

\subsection{Agent-based and evolutionary perspectives}

An important aspect that has attracted the attention of scholars is the emergence, and in general the evolution, of norms. Several authors have analyzed the 


\begin{tabular}{lcc} 
Cohort & Lower age limit & Upper age limit \\
\hline $1945-47$ & $71 \%$ & $11 \%$ \\
$1959-61$ & $66 \%$ & $6 \%$ \\
$1973-73$ & $58 \%$ & $5 \%$
\end{tabular}

Table 1: Percentage of women who agree on the existence of an age before which or after which one should not enter unions

issue, especially in a game-theoretical context (see for instance [2]), in which the existence of social norms may explain some of the paradoxes of rational-choice theory. Recently, Bendor and Swistak [1] have shown that the existence of norms, and in particular of social norms (with enforcement of behavior from a third person), for a very general type of 2-person games can be justified from the point of view of evolutionary game theory. For our purposes, it is important to justify the long-term persistence of norms that restrict life-course choices, and that are apparently counter-productive as far as reproductive success is concerned. Social influence may have an important role in reinforcing the dynamics of norms, as it is generally true for demographic choices [20].

There has also been growing interest in the study of social norms within the field of agent-based modelling [11], [10]. Designing agents who might follow social norms calls for having a more and more precise definition of norms. In particular, they call for an explicit study of how norms are represented in agents' mind, a problem which has a key relevance in empirical studies. In the agentbased modelling literature, it has been argued that norms can be implemented in simulation as built-in constraints, as built-in ends (goals), or as built-in obligations [6], [24]. Agent-based modelling is also a natural toolkit for the study of the evolution of norms on demographic events. It gives the possibility to study the existence of long-term situations (in some cases, long-term equilibria) where one or more norms co-exist in a population [23]. The evolution of norms, which has been a key topic in the literature, may be particularly interesting when such norms concern behaviors directly affecting population dynamics. Conversely, 
given that such norms deal with demographic behavior, and then naturally affect the evolution of a population of agents, they provide a special case-study for scholars interested in the evolution of norms in agent-based simulation [4]. ${ }^{1}$

We use a very simple definition of norm: a constraint built in individuals. We assume that individuals can marry only within a specific age interval, and that such an interval cannot change during the course of their life. The age interval, so, constitutes an age-at-marriage proscriptive norm. The use of a norm as a built-in constraint does not necessitate for the explicit consideration of sanctions - the sanctions are so strict that it is not possible at all to overcome the normative age interval ${ }^{2}$. Individuals can only marry other individuals with a compatible norm. We can see norms and mechanisms of transmission of norms as memes, although this is not strictly necessary. ${ }^{3}$

\section{An agent-based model}

We develop an agent-based model to simulate the dynamics of norms addressing in particular 1) their long-term persistence or their disappearance; 2) the longterm impact of the initial distribution of norms in a population; 3) the impact of random mutation and social influence. This section provides an outline of the model. We assume a population of individuals with a given, fixed population size

$s$. Each individual is characterized by an age-at-marriage proscriptive norm (we simply call it either norm or interval from now on). Each norm $i$ is represented by

\footnotetext{
${ }^{1} \mathrm{~A}$ population of agents does not necessarily have to be a population of normative agents: agents can be thought as being diverse in their rationality [8]. This fits very well with the idea that decisions underpinning demographic events may be based on different types of rationality. In particular, following a social norm may just be one of the possible ways of acting. How this applies when social norms concern demographic events, together with its possible consequences on population dynamics is also a specifically interesting problem. In this paper, however, we will concentrate on a purely normative population.

${ }^{2}$ In fact, the built-in-constraint idea corresponds to assuming an infinite cost for the violation of norms.

${ }^{3}$ The idea of studying the evolution of norms as memes [12] has been recently also adopted by $[15]$.
} 
a lower acceptable limit of the age at marriage, $l_{i}$, and by an upper acceptable limit of the age at marriage marriage $u_{i}$. The norms for the first generation $G_{0}$ of individuals are initialized randomly. More specifically, each individual's norm is initialized by first picking a lower bound according to a user-specified random distribution. Then, an upper bound is chosen by repeatedly drawing from another random distribution until a value larger than the lower bound is found. ${ }^{4}$ Alternatively, the user may also specify a distribution for the lengths of the interval for acceptable ages. In all cases, values can be drawn from any arbitrary distribution we choose to define. The options that we consider in what follows include (a) constant values (in case of specifying the length of the interval); (b) uniform random distributions; (c) normal distributions, and (d) bimodal distributions.

Starting from the initial generation $G_{0}$, we simulate the evolution of the experimental population forward in time, resulting in a sequence of generations $G_{t}, t=0, \ldots, T$ with $T$ indicating the number of generations to be simulated. In particular, we (a) allow individuals to marry each other; (b) allow the procreation of children within each generation, and (c) define a mechanism governing the transmission of norms.

For marriage, an individual $i$ has to find a partner $j$ for which $\left[l_{i}, u_{i}\right] \cap$ $\left[l_{j}, u_{j}\right] \neq 0$ holds. More specifically - after randomizing the order of the individuals in the current generation - we start with the first individual and search for the first acceptable partner (such that both age intervals overlap). In case such a pair of individuals cannot be found, the first individual remains single and is

\footnotetext{
${ }^{4}$ This procedure does not warrant a uniform distribution for both lower and upper bounds. If, for example, both lower and upper bounds are specified to be drawn from a uniform distribution, only the lower bound will actually be uniformly distributed, while the distribution for upper bounds will be more concentrated on for higher ages (the higher the age the larger is the number of lower bounds for which this age is a valid upper bound). We could not find a trivial way of fixing this problem (e.g., a similar procedure where an invalid pair is simply discarded would lead to an analogue over-sampling of lower bounds). However, we do not think that this is a crucial problem in the current model.
} 
no longer considered, otherwise both partners marry and are removed from the candidates list. Then, the same search algorithm is applied to all subsequent individuals. In the model presented here, although age is used as a descriptor of the norms, it is not a characteristics of agents themselves. Agents do not age during their search for a marriage partner or reproduction: they are born, marry, reproduce, and die during the space of one generation.

Procreation only takes place among those individuals who have found a partner. We introduce a simplifying assumption here: the population is stationary, of size $s$. This is another demographic weakness of the model we present here: reproductive success does not depend on age at marriage. To assure that the population is stationary in each subsequent generation, we proceed as follows: we first assign $\min \left(\left\lfloor\frac{s}{c}\right\rfloor, k\right)$ children to each married couple, where $c$ is the number of couples found, and $k \geq 0$ is a parameter set by the user, which specifies the minimum number of kids a couple should have. Then, the generation is filled up by successively drawing, with replacement, couples from the set of married couples, and by assigning one child for each pick until the generation size $s$ has been attained. Thus, setting the parameter $k$ to a number $\geq\left\lfloor\frac{s}{c}\right\rfloor$ will ensure that the distribution of $s$ children among the $c$ couples is as uniform as possible. On the other hand, if $k=0$, the $s$ children of the next generation are determined by randomly assigning each of them to one of the $c$ couples in the parent generation. In this case some couples may remain childless.

Central to the evolution of age norms on marriage is the mechanism of intergenerational transmission of the parents' norms. Here we allow for four different transmission mechanisms of the parents' norms $\left[l_{p 1}, u_{p 1}\right]$ and $\left[l_{p 2}, u_{p 2}\right]$ to their child's norms $\left[l_{c}, u_{c}\right]$ :

Intersection: The child's norm is the intersection of the age interval of its 
parents.

$$
l_{c}=\max \left(l_{p 1}, l_{p 2}\right), u_{c}=\min \left(u_{p 1}, u_{p 2}\right)
$$

Union: The child's norm is the union of the age interval of its parents.

$$
l_{c}=\min \left(l_{p 1}, l_{p 2}\right), u_{c}=\max \left(u_{p 1}, u_{p 2}\right)
$$

Random: The bounds of the child's age norm are randomly chosen from the respective bounds of the parent's age norm.

$$
l_{c}=\operatorname{random}\left(l_{p 1}, l_{p 2}\right), u_{c}=\operatorname{random}\left(u_{p 1}, u_{p 2}\right)
$$

(random $(x, y)$ picks either $x$ or $y$ with equal probability.)

Uniform: The lower bound (respectively upper bound) of the child's age norm is drawn from a uniform distribution between the lower bounds (respectively upper bounds) of the parents' age norms.

$$
l_{c}=\operatorname{uniform}\left(l_{p 1}, l_{p 2}\right), u_{c}=\operatorname{uniform}\left(u_{p 1}, u_{p 2}\right)
$$

(uniform $(x, y)$ picks a number $r$ with $x \leq r \leq y$ with uniform probability.)

To allow for more than one intergenerational transmission mechanism, we choose the following implementation. Besides the age norm, individuals are assigned a specific transmission mechanism. For the first generation $G_{0}$, we assume an exogenous probability with which each individual is assigned one transmission mechanism out of the four defined above. For example, we could assign all four mechanisms with probability 0.25 each, or we could assume the existence of the intersection and union mechanism only assigning them with 
probability 0.5 each ${ }^{5}$. The transmission mechanism does not have an influence on the choice of partners, but children inherit the transmission mechanism from one of their parents. In the case of conflicts (the parents have different transmission mechanisms), one is selected at random (with equal probabilities). The selected transmission procedure, which will be inherited to the child, is also the one that is used to compute the child's age norm from its parents' norms.

In addition to the intergenerational transmission of age norms, we also allow for two alternative kinds of mutations at a user specifiable rate $m, 0 \leq m \leq 1$. This means, with a probability of $m$, a child does not inherit any information from its parents (neither age norms nor the transmission procedure) but instead will be a mutant. We implement the following alternative mutations. (1) A child may be initialized randomly in the same way as the individuals of the first generation. (2) The child's lower and upper bound on the age norm are set equal to the mean value of all lower and upper bounds in the parent generation, and its transmission mechanism is set to the most frequent transmission mechanism in the parent generation. This mutation mechanism is built to embed in the evolution of our population the possibility of social influence, which is a crucial mechanism for the evolution of norms.

The assumptions we make in building the model are, of course, highly abstract. They however basically correspond to similar assumptions introduced by scholars concerned with the evolution of cultural traits, and norms on ageat-marriage can be seen as an important cultural trait. On the one hand, many cultural traits are intergenerationally transmitted (see i.e. [7]). On the other hand, intergenerational transmission (as well as the adoption of a the trait of another random individual) produce only what has been defined unbiased transmission. The mutation mechanism we built in the model also account for a social

\footnotetext{
${ }^{5}$ We could, of course, also use skewed probability distributions where one transmission mechanism dominates the others. This is another issue that we have not yet systematically studied.
} 
feed-back in the form of conformist transmission, where the most frequent trait in the preceding generation is transmitted to the next generation (see i.e. [5], [18]). This kind of transmission allows to stress once again the importance of social interactions in shaping demographic behavior [20].

\section{Results and discussion}

The agent-based model we introduced in the previous section has been implemented in Lisp-Stat[26], and it can be used to conduct various experiments. We first study the conditions that lead to the long-term persistence of norms (with the possibility of having sub-populations with different norms) or to the dissolution of norms (considered as the widening of the acceptable age interval to the maximum length). Then, we analyze the impact of initial conditions on the dynamics of norms. Finally, we study mutation mechanisms, including the impact of social influence on the dynamics of the process.

\subsection{Long-term persistence or dissolution?}

We start to investigate whether and which transmission mechanisms allow for the long-term persistence or dissolution of norms. For this set of experiments we set the population size to 1,000 and we initialize the age norm of the first generation by picking a lower and upper number randomly from the uniform interval $[16,60]$. Moreover we set the mutation rate to zero and place no restrictions $(k=0)$ on the number of kids per parents (i.e., a couple may have an arbitrary number of children, including zero). Thus, we operate in a setting of pure intergenerational transmission. We then alternatively apply each of the four transmission mechanisms described in the previous section and plot for selected generations the resulting age norms of all agents (Figure 1).

Assuming that child's norm is the intersection of the age interval of its 
(a) intersection combiner
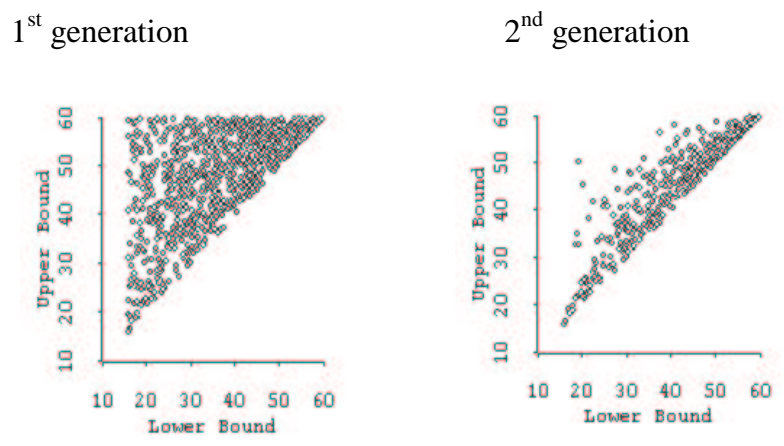

$$
5^{\text {th }} \text { generation }
$$

$100^{\text {th }}$ generation

$$
200^{\text {th }} \text { generation }
$$
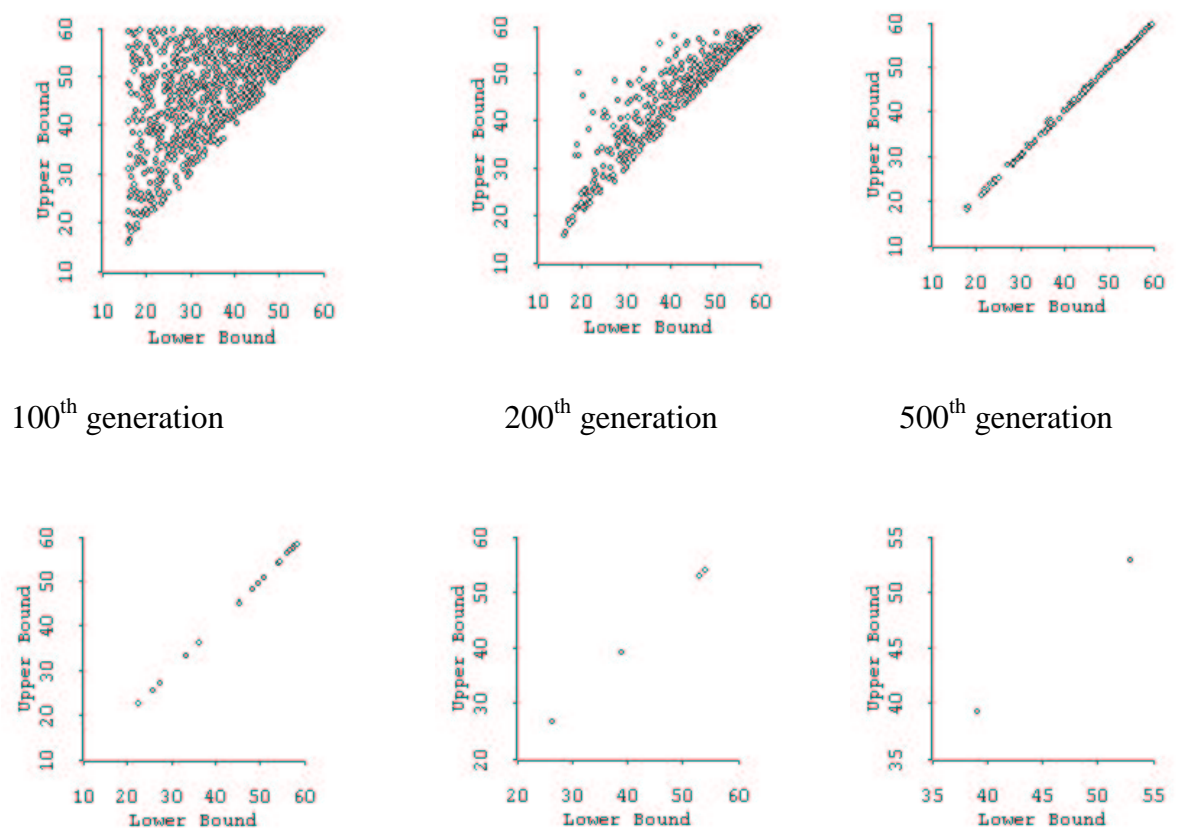

(b) union combiner

$1^{\text {st }}$ generation

$$
5^{\text {th }} \text { generation }
$$

$100^{\text {th }}$ generation
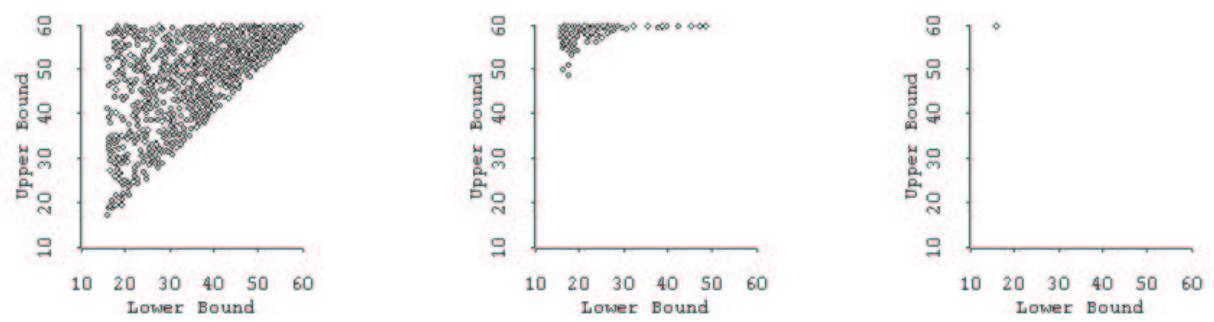

Figure 1: Evolution of age-at-marriage norms for various transmission mechanisms. Each circle represents an observed combination of lower and upper bound for the acceptable age interval. 
(c) intersection and union combiner

$1^{\text {st }}$ generation

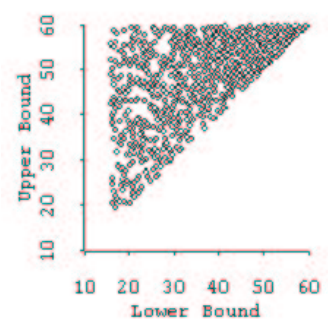

(d) random combiner

$1^{\text {st }}$ generation

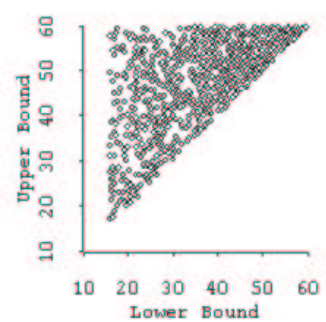

(e) uniform combiner

$1^{\text {st }}$ generation

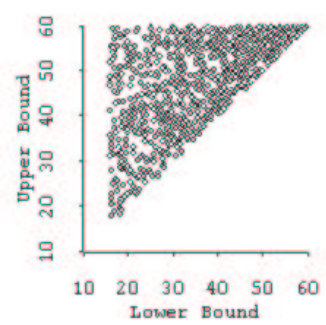

$5^{\text {th }}$ generation

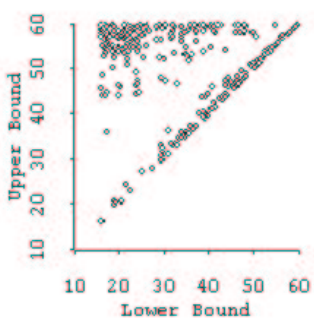

$5^{\text {th }}$ generation

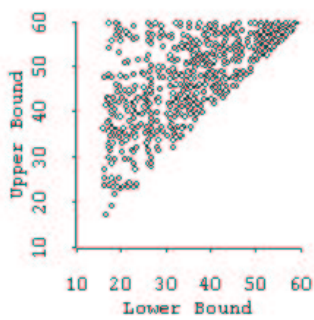

$5^{\text {th }}$ generation

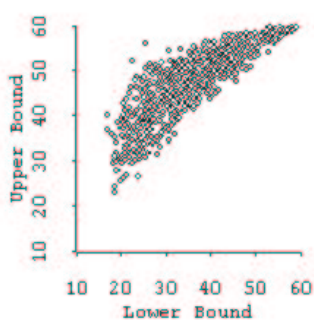

$100^{\text {th }}$ generation

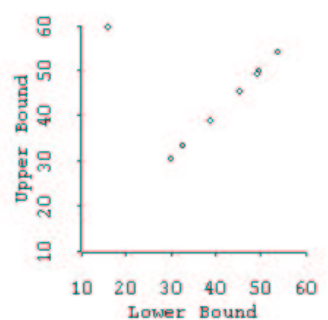

$100^{\text {th }}$ generation

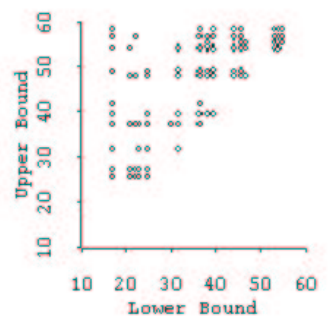

$100^{\text {th }}$ generation

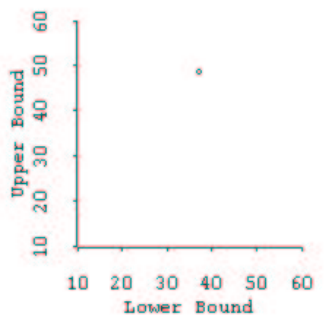

Figure 1: Evolution of age-at-marriage norms for various transmission mechanisms. Each circle represents an observed combination of lower and upper bound for the acceptable age interval. 
parents (Figure 1.a) we observe a convergence of norms towards the diagonal already during the first few generations. Eventually, only a few norms with very short length survive (e.g., only two at generation 500), each of them dominating a group of agents who are only able to marry among themselves. Plotting the mean lengths of intervals and the number of couples versus the number of the generation, Figure 2.a demonstrates the fast convergence towards age norms that are characterized by very narrow age intervals, but that the number of couples that marry within each generation is constantly increasing (after a sharp decline in the beginning). This reflects the fact that the narrow age intervals are shared among more and more individuals so that less and less subjects do not find a partner. Although the results of this experiment are easily predictable, we prefer to present them to have an easily understandable point of comparison for the experiments that follow.

If we assume that the child's norm is the union of the age interval of its parents, we observe a rapid dissolution of norms. As Figure 1.b and 2.b evidence, the mean length of intervals rapidly converges towards the maximum possible length of 44 years and the number of couples formed reaches its maximum of 500 already during the first few generations of the simulation.

Assuming that an individual of the initial generation is randomly assigned either the intersection or the union transmission mechanism (with equal probabilities), Figure 1.c and Figure 2.c show the possibility of the diversity of rationalities: About half of the population has no norm, converging to a norm of the maximal length 44 (upper left corner), while other parts converge to a binding short-length norm at the diagonal. Consequently, the mean length of intervals fluctuates around 22 years.

If the lower and upper bound of a child's norm are randomly selected by picking one of the respective bounds of its parents, we observe convergence 
Mean length

(a) intersection combiner

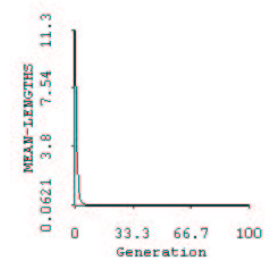

(b) union combiner

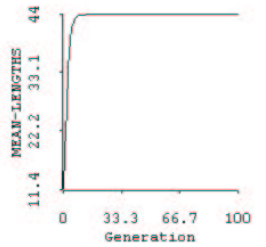

(c) intersection and union combiner

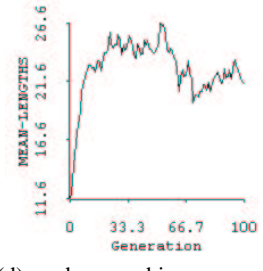

(d) random combiner

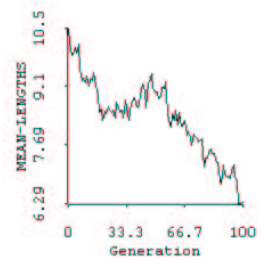

(e) uniform-combiner

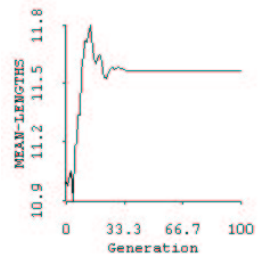

Number of couples
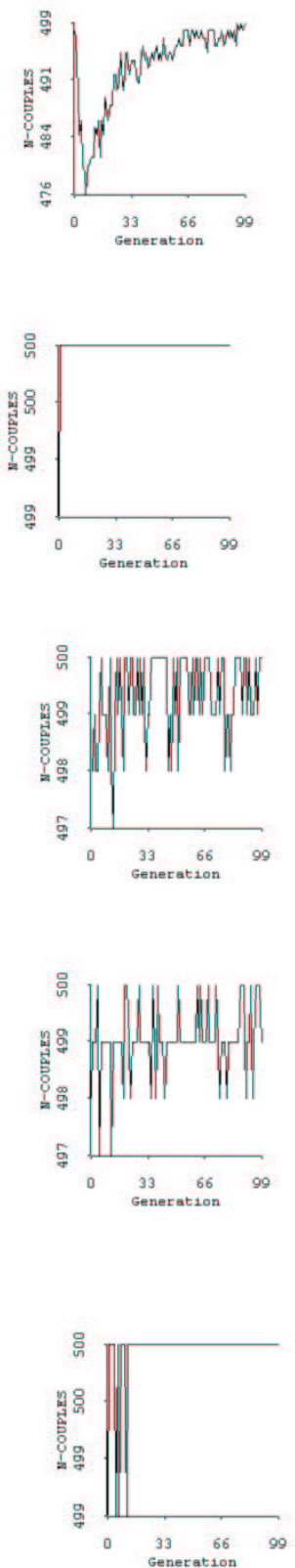

Figure 2: Mean length of age norm and number of couples formed for the first 100 generations applying alternative transmission mechanisms as given in Figure 1. 
towards a few selected lower and upper bounds, which are scattered more or less evenly (but not densely) throughout the range of possible values (Figure 1.d). The ordered appearance of the points on certain lines is due to the fact that no new norms enter the norm pool after the first generation. Some of the bounds will eventually die out because the individuals that use them are unfortunate enough not to find a partner, not to have children, or to pass their partners' bound to their children (and not their own). On the other hand, the fewer norms there are in the norm pool, the more individuals share these preferences and the less likely it becomes that a certain norm dies out. Consequently, the pattern becomes increasingly less dense over the generations, but the speed at which norms vanish decreases. Nevertheless, the population is not yet stable at generation 100, as can be seen from the decrease in the average length of the norms.

Finally, we consider the case where the lower/upper bound of a child's norm is selected by picking values randomly between the upper/lower bounds of its parents. We observe a continuous and relatively fast contraction of the population towards a single point. The reason for this phenomenon is simply that extreme values for bounds (values near the maximum or minimum value that occurs within the generation) will die out because they are likely to be paired with a partner that has a value that is further away from that bound, and hence its children are likely to inherit a shorter age interval. The attractor point is near $38 / 49$, which is explained by the fact that 38 is the mean value for the lower bound in the initial generation, and 49 would be the mean value between 38 and 60 (remember that upper bounds are drawn repeatedly until we draw a value that is larger than the previously drawn lower bound).

To investigate the role of the fertility distribution in the dissolution or emergence of norms we run an experiment where we set the minimum number of kids 
$1^{\text {st }}$ generation

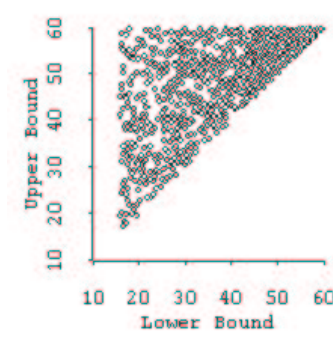

$100^{\text {th }}$ generation

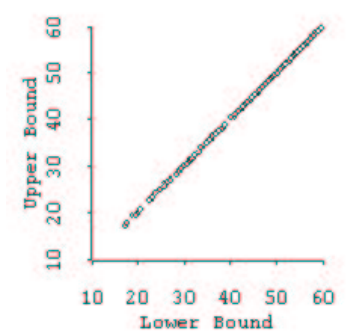

$2^{\text {nd }}$ generation

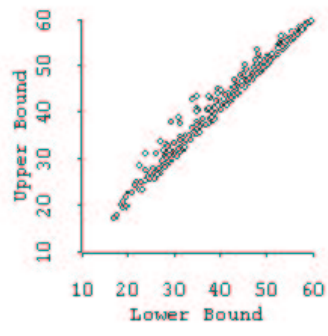

$200^{\text {th }}$ generation

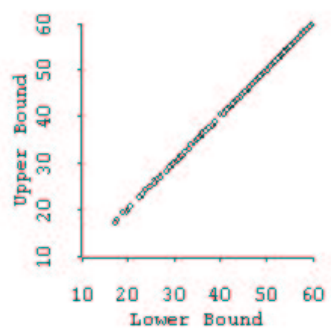

$5^{\text {th }}$ generation

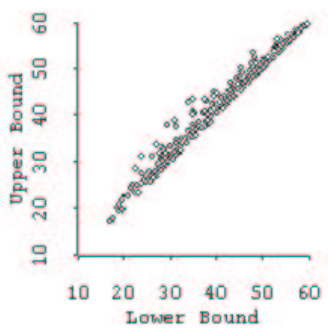

$500^{\text {th }}$ generation

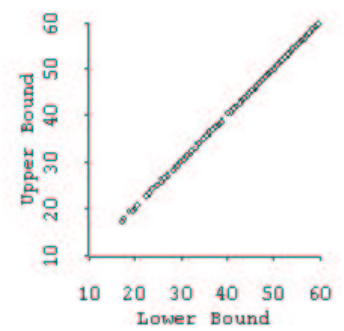

Figure 3: Evolution of age-at-marriage norms applying the intersection mechanism and setting the minimum number of kids equal to two. Each circle represents an observed combination of lower and upper bound for the acceptable age interval.

equal to two, i.e., the parameter $k=2$ in the agent-based model of section 3 . In Figure 3, we show the results for the same setting as in Figure 1.a, i.e., for the case of intersection as the norm transmitter. The results show that if fertility is more evenly spread among the agents, which is the case if we assume that $k=2$, we observe that the intersection mechanisms will not result in the emergence of only one or two norms. In fact, the norms line up densely along the diagonal. This can be explained by the fact that if each couple has at least two children, it is less likely that any norm will disappear, since there will always be children that transmit the age norm of their parents. A similar argument would hold in case of the experiment in Figure 1.c and Figure 1.d. if we would set $k=2$. I.e. the number of norms that will disappear would decrease if fertility is more 
Intersection combiner

(a)

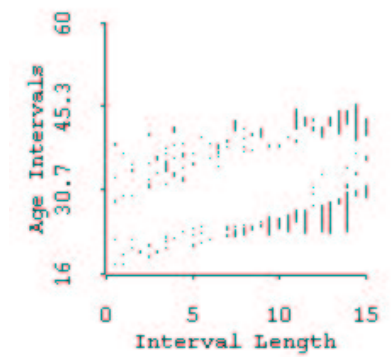

(b)

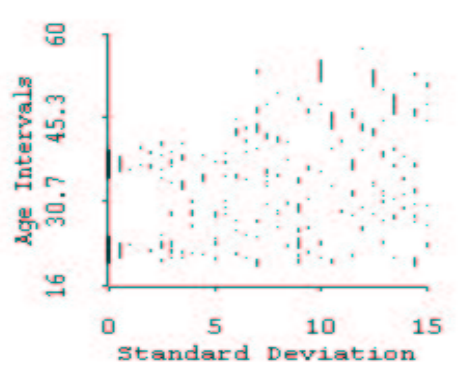

Union combiner
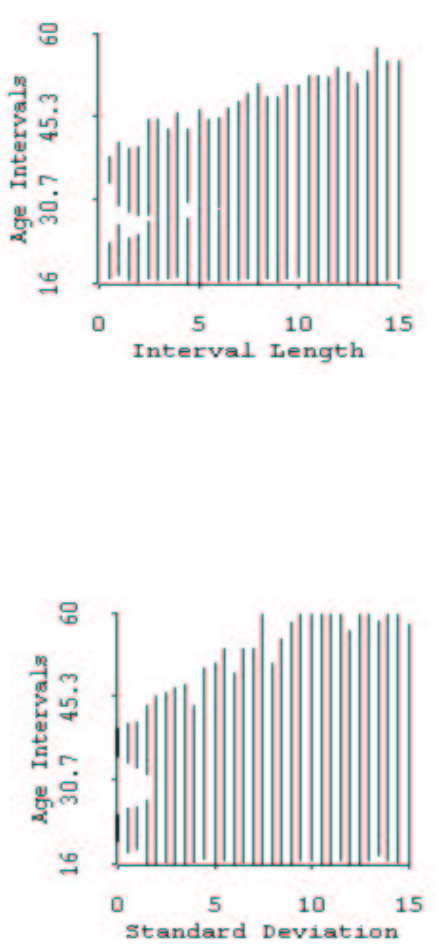

Figure 4: Observed age norms in the 10th generation plotted on top of each other versus the assumed interval length in the initial generation (a) and versus the standard deviation of the bimodal distribution in the initial generation (b).

evenly spread among the agents.

\subsection{The long-term impact of initial conditions}

In our second experiment, we are interested in whether the initial distribution of norms has an impact on the long-term configuration of norms. For this experiment we initialize the first generation with 100 individuals. We then initialize the age norm of the first generation by assuming a bimodal normal 
distribution with means 20 and 35 and standard deviations 2 and 3 for the lower bounds and assume that all norms have the same fixed length (i.e., the upper bound is determined deterministically from the lower bound). We performed a series of experiments in which the length of this interval varied from 0 to 15 years. Again, the minimum lower bound is set to age 16 and the maximum upper bound to age 60 (i.e., if the bimodal random function returns a value that is out of these bounds, we draw another value). We then apply either the intersection or union transmission mechanism setting the mutation rate and the parameter $k$ equal to zero (again, this is a setting with pure intergenerational transmission). We chose a bimodal initial distribution to stress the diversity between groups (two groups in our case) in the initial generation. In particular, here our interest is somehow similar to that of studies concerned with the emergence and long-term persistence of between-group differences (see i.e. [18]). Do initially different groups maintain their diversity in the long run?

To visualize whether the initial diversity persists, we plot all age norms in the 10th generation of the entire population versus the assumed interval length (Figure 4.a). In other words, a point is black if the corresponding age is part of at least one age interval in the generation, and white if it does not appear in any norm interval. Figure 4.b shows the results of a similar experiment, where we fixed the length of the age interval to 5 , and varied both standard deviations of the bimodal distribution simultaneously (both are set to the same value between 0 and 15).

In case of the intersection transmission mechanism we observe a persistence of the initial distribution of age norms, i.e. a distinction between two groups of age norms. On the other hand, applying a union transmission mechanism shows that the initial bimodal distribution of the age norms does not have any long-term impact except for very short interval lengths. That is, in this simple 
experiments, diversity persists only if groups are significantly separated at the beginning of the process.

\subsection{Random mutations and social influence}

In a further experiment we are interested in the influence of 1) random mutations and 2) social influence on the evolution of age-at-marriage norms. Let us recall that both mechanisms are implemented as mutations (i.e., deviations from pure intergenerational transmission) in our model. For the sake of simplicity, we assume the same initialization as in Figure 1 a (i.e., we focus only on the intersection transmission mechanism) except that we allow for mutations in each generation.

Let us start with purely random mutations (i.e., mutation that pick individual characteristics randomly from the same distribution used for generation 0 ). We plot the resulting distribution of age norms for the 100th generation for various rates of mutation. As these results show (Figure 5), a random mutation is not able to hamper the persistence of norms. Depending on the rate of random mutations, we observe that a specific percentage of individuals deviates from age norms that are lined up along the diagonal and are representative of the persistence of norms. However, the clear majority of the points still converges towards zero-length points on the diagonal.

We end our set of experiments by referring to the role of social influence in the evolution of marriage norms. Again we assume the same initialization as in Figure 1 except that we now allow for 'mean mutation' in each generation. That is, for those individual who have a mutation, age-at-marriage norms are derived using the mean upper and lower intervals of the parents' generation. This introduces what has been defined a conformity bias [5], [18], a tendency of individuals to conform to the most commonly encountered behavior, and formal- 
$1^{\text {st }}$ generation

(a) mutation rate $=0.01$

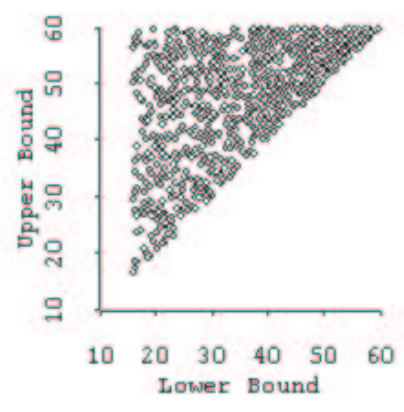

(b) mutation rate $=0.1$

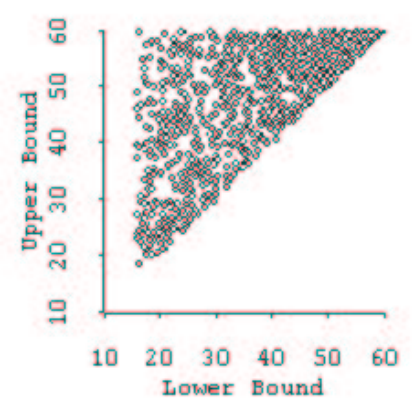

(c) mutation rate $=0.3$

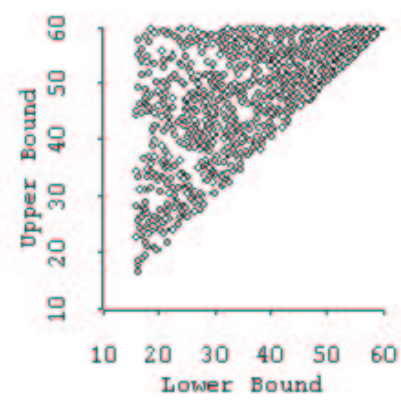

$100^{\text {th }}$ generation
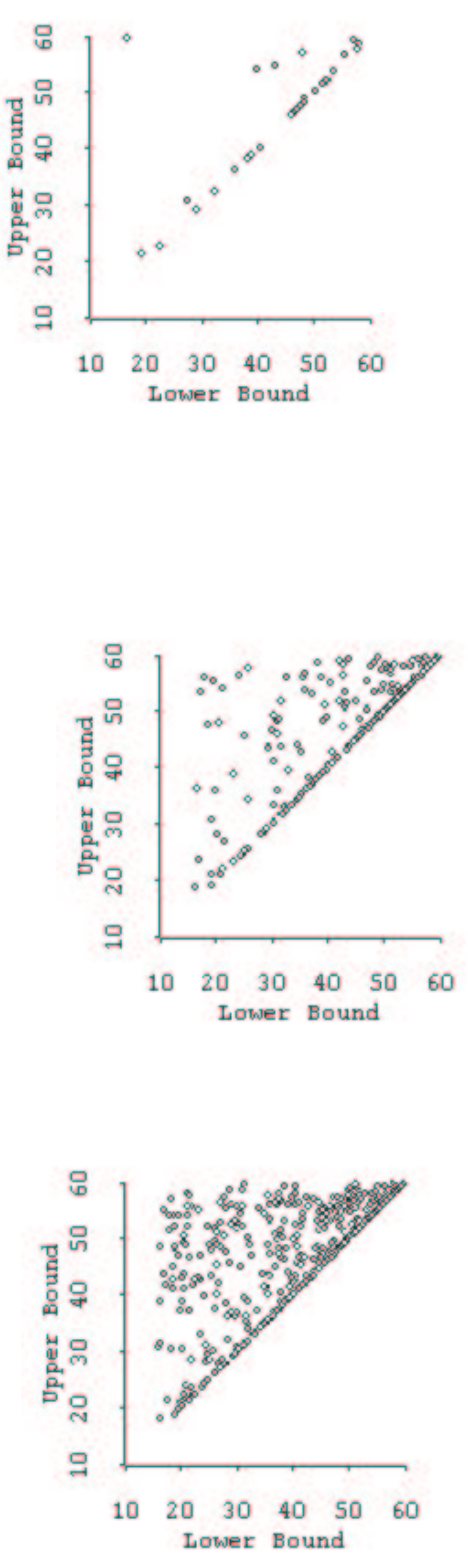

Figure 5: Persistence of age-at-marriage norms under various degrees of random mutation assuming an intersection transmission mechanism. Each circle represents an observed combination of lower and upper bound for the acceptable age interval. 
$1^{\text {st }}$ generation

(a) mutation rate $=0.5$, intersect combiner

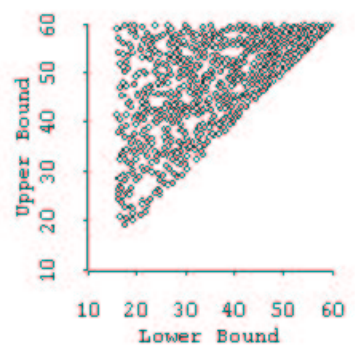

(b) mutation rate $=0.9$, union combiner

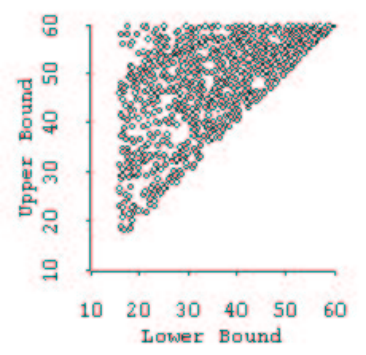

$100^{\text {th }}$ generation
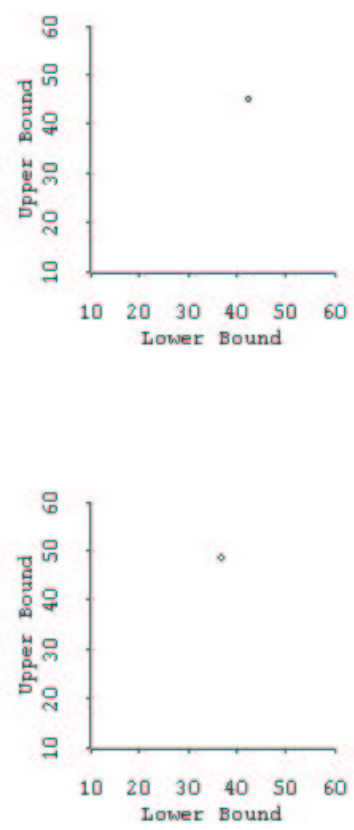

Figure 6: The effect of social interaction on the evolution of age-at-marriage norms assuming an intersection and alternatively a union transmission mechanism. Each circle represents an observed combination of lower and upper bound for the acceptable age interval.

izes the importance of social influence. To illustrate the effect of such factor, we apply the intersection and the union transmission mechanism of norms (Figure 6.a and 6.b respectively). As the results indicate, the presence of social influence implies a faster convergence towards the emergence of societal-level norms in case we assume the intersection transmission mechanism. However, the presence of social influence may also result in a reduction of the speed of dissolution of norms in the case where we impose the union transmission mechanism. In Figure 6.b., we observe that the final age norm has a smaller interval length 
than in the experiment in Figure 1.b, where we excluded social influence. Due to social influence, the point of convergence shifts towards the mean values of the lower and upper bounds respectively. Note that the point of convergence is quite similar to the one obtained with the uniform combiner (Figure 1.e), which also has a tendency to converge towards this interval, although the pattern of convergence is different in both cases. Our experiments show that including social influence is a powerful mechanism in the evolution of norms, norms such as the one we model may persist under a relatively broad range of conditions ${ }^{6}$.

\section{Summary and prospects for future research}

In this paper, we introduced a highly stylized agent-based model on the evolution of age-at-marriage norms. We used a very simple definition of norm as agents' built-in-constraint. We showed that, under particular assumptions on the intergenerational transmission of norms, such norms may persist in the long-run. This simple model also allowed for cases of diversity of norms (subpopulations following different norms), as well as for diversity of rationality (part of the population following a norm and part of the population without constraint). The impact of the initial distribution was also shown to matter in specific cases, with threshold effects. Social influence, also incorporated in this model, significantly modified the speed of evolution of the process.

We believe that agent-based modelling can give a sound contribution to the theory of life-course norms. Nevertheless, it is clear that in order to develop a more realistic model of the evolution of life-course norms much further work is needed. In this sense, this paper gives less answers than it opens questions. Let us mention some of these questions. For one, we should complement the

\footnotetext{
${ }^{6}$ Henrich and Boyd [18] showed that under a broad range of environmental conditions, conformist transmission explains the maintenance of cultural difference between groups.
} 
presented work with a systematic study of the impact of different initial distributions of the norms, a facility that is foreseen in the current version of the program, but to which we have not paid enough attention yet. Furthermore, we cannot foresee whether the long-term persistence of constraints on life courses can be confirmed in a less restrictive model. This will be a question for future research. Moreover, as emphasized in the agent-based modelling literature, norms as built-in-constraint are a special case. Normative choice, that is the choice to behave (or not to behave) according to norms, is the general framework in the direction of which future research may move. A simple extension of the model would be to use less restrictive forms of norms (probability distributions instead of fixed-length intervals).

The realism of the demographic part of our agent-based model needs also to be improved. First, in the current setup, the age of agents is not relevant, while clearly it is in the real world. Explicitly introducing age would increase significantly the complexity of the model, but it will also reveal more in depth the specificity of age-at-marriage norms with respect to other kinds of norms. Second, age at marriage and fertility are independent. Fertility and length between generations, as it is in real life, are a function of the age at marriage, and this may influence the evolution of age-at-marriage norms in a very important way. Third, the present model is basically a one-sex model. This is problematic as from a marriage market perspective, age-at-marriage norms may change in situations of unbalanced ratios between sexes (which may arise if we really consider the agents as they age). Finally, mobility and spatial segregation may also act as important factors in maintaining separate cultures (see i.e. [14]). Further research on these issues is undoubtedly necessary. 


\section{Acknowledgments}

We would like to acknowledge suggestions from the participants of the workshop on Agent-Based Computational Demography (Max Planck Institute for Demographic Research, Rostock, February 2001). We thank Arnstein Aassve, Hans-Peter Kohler, and especially Peter Todd for comments which helped us to significantly improve this paper. Of course, we are entirely responsible for all remaining weaknesses. A final disclaimer: views expressed in this paper are attributable to the authors and do not necessarily reflect those of the institutions they belong to.

\section{References}

[1] Bendor J., Swistak P., 2001, "The Evolution of Norms", American Journal of Sociology, 106, 6: 1493-1545.

[2] Bicchieri C., Jeffrey R., Skyrms B. (Eds.), 1997, The dynamics of norms, Cambridge University Press, Cambridge.

[3] Billari F.C., Micheli G.A., 1999, "Le scelte demografiche: la percezione dei costi e delle norme sociali", in Mauri L., Billari F.C. (Eds.), Generazioni di donne a confronto. Indagine sociodemografica, FrancoAngeli, Milano.

[4] Billari F.C., Micheli G.A., 2001, "Social norms on agents' demographic events. Preliminaries for a (multi-)agent-based approach", in Conte R., Dellarocas C., Social Order in Multiagent System, Kluwer, Dordrecht.

[5] Boyd R., Richerson P.J., 1985, Culture and the Evolutionary Process, The University of Chicago Press, Chicago.

[6] Castelfranchi C., Conte R., Paolucci M., 1998, "Normative reputation and the cost of compliance", Journal of Artificial Societies and Social Simulation, 1, 3, http://www.soc.surrey.ac.uk/JASSS/1/3/3.html.

[7] Cavalli-Sforza L.L., Feldman M.W., 1981, Cultural Transmission and Evolution: A Quantitative Approach, Princeton, Princeton University Press.

[8] Conte R., 1997, "Diversity in rationality. A multi-agent perspective", Paper given at the Dagstuhl Seminar on Social Science Microsimulation, May 5-9.

[9] Conte R., Castelfranchi C., 1995, Cognitive and social action, London, UCL Press. 
[10] Conte R., Castelfranchi C., 1999, "From conventions to prescriptions. Towards and integrated view of norms", Artificial Intelligence and Law, 7: 323-340.

[11] Conte R., Falcone R., Sartor G., 1999, "Introduction: Agents and Norms: How to fill the gap?", Artificial Intelligence and Law, 7: 1-15.

[12] Dawkins R., 1976, The Selfish Gene, Oxford, Oxford University Press.

[13] Epstein J.M., 2001, "Learning to be Thoughtless: Social Norms and Individual Computation", Computational Economics, 18: 9-24.

[14] Epstein J.M., Axtell R., 1996, Growing Artificial Societies. Social Science from the Bottom Up, Brookings Institutions Press, Washington, D.C.

[15] Flentge F., Polani D., Uthmann T., 2001, "Modelling the Emergence of Possession Norms using Memes", Journal of Artificial Societies and Social Simulation, 4, 4, http://www.soc.surrey.ac.uk/JASSS/4/4/3.html.

[16] Giele J.Z., Elder G.H.Jr. (Eds.), 1998, Methods of Life Course Research. Qualitative and Quantitative Approaches, Thousand Oaks, CA, Sage.

[17] Heckhausen J., 1999, Developmental Regulation in Adulthood. AgeNormative and Sociostructural Constraints as Adaptive Challenges, Cambridge, Cambridge University Press.

[18] Henrich J., Boyd R., 1998, "The Evolution of Conformist Transmission and the Emergence of Between-Group Differences", Evolution and Human Behavior, 19: 215-241.

[19] Horne C., 2000, "Community and the State. The Relationship between Normative and Legal Controls", European Sociological Review, 16: 225243.

[20] Kohler, H.-P., 2001, Fertility and Social Interaction. An Economic Perspective, Oxford, Oxford University Press.

[21] Livi Bacci, H.-P., 2000, The Population of Europe, London, Blackwell.

[22] Marini M.M., 1984, "Age and sequencing norms in the transition to adulthood", Social Forces, 63: 229-44.

[23] McElreath R., Boyd R., Richerson P.J., forthcoming, "Shared norms can lead to the evolution of ethnic markers", Current Anthropology.

[24] Saam N.J., Harrer A., 1999, "Simulating Norms, Social Inequality, and Functional Change in Artificial Societies", Journal of Artificial Societies and Social Simulation, 2, 1, http://www.soc.surrey.ac.uk/JASSS/2/1/ 2.html. 
[25] Settersten R.A., Hägestad G.O., 1996, "What's the Latest? Cultural Age Deadlines for Family Transitions", The Gerontologist, 36: 178-188.

[26] Tierney L., 1990, Lisp-Stat. An Object-Oriented Environment for Statistical Computing and Dynamic Graphics, New York, Wiley.

[27] White J.M., 1998, "The Normative Interpretation of Life Course Event Histories", Marriage \& Family Review, 27: 211-235.

[28] Wilson C., 1999, "Evolutionary Theory and Historical Fertility Change", Population and Development Review, 25: 531-541. 